\title{
BMJ Open Evaluation of the implementation of information system for postdischarge with the theoretical domains framework by healthcare professionals: a multistage design with qualitative inquiry and Delphi expert discussion protocol
}

\author{
Eliza LY Wong (D) , ${ }^{1}$ KS Tang, ${ }^{2}$ Dong Dong, ${ }^{1}$ Phoenix KH Mo, ${ }^{1}$ Annie WL Cheung, ${ }^{1}$ \\ Jack CH Lau, ${ }^{1}$ EK Yeoh ${ }^{1}$
}

To cite: Wong ELY, Tang KS, Dong $\mathrm{D}$, et al. Evaluation of the implementation of information system for postdischarge with the theoretical domains framework by healthcare professionals: a multistage design with qualitative inquiry and Delphi expert discussion protocol. BMJ Open 2021;11:e046081. doi:10.1136/ bmjopen-2020-046081

- Prepublication history and additional supplemental material for this paper are available online. To view these files, please visit the journal online (http://dx.doi.org/10.1136/ bmjopen-2020-046081).

Received 20 0ctober 2020 Accepted 18 0ctober 202

A Check for updates

(c) Author(s) (or their employer(s)) 2021. Re-use permitted under CC BY-NC. No commercial re-use. See rights and permissions. Published by BMJ.

${ }^{1}$ Centre for Health Systems and Policy Research, JC School of Public Health and Primary Care, Faculty of Medicine, The Chinese University of Hong Kong, Hong Kong, China

${ }^{2}$ Hong Kong Hospital Authority, Hong Kong, China

Correspondence to Professor Eliza LY Wong; lywong@cuhk.edu.hk

\section{ABSTRACT}

Introduction Improving discharge information dissemination may improve patients' health literacy of self-care and health outcomes, avoid unnecessary healthcare utilisation, and reduce the healthcare cost. This study aims to use an implementation science theory guided approach to examine the beliefs and behaviours of healthcare professionals regarding postdischarge information summary (PDIS) implementation in a public inpatient setting.

Methods and analysis A multistage study design involving qualitative inquiry and Delphi expert discussion will be used to systematically explore the perceived barriers in the four implemented hospitals and enable the full implementation of the PDIS in geriatric and medical care. The theoretical domains framework (TDF), behavioural change wheel and realistic evaluation framework will be used to guide the investigation of implementation. This study consists of three steps: (1) identifying barriers and enablers from an implementation perspective using a TDF-informed interview guide; (2) devising theory-based implementation strategy packages to facilitate the adoption and enhancement of PDIS by performing a strategy mapping exercise and (3) developing an effective implementation strategy package for scaling up PDIS in other target hospitals as well as other specialities using the Delphi expert discussion. The goal of this multistage study design is to identify the perspectives from healthcare professionals towards the PDIS implementation and explore their barriers and facilitators of the process in the pilot phase. The invited healthcare professionals would share their daily experience on providing PDIS to patient in various study hospitals with similar ward setting. The implementation of discharge intervention in a study setting through different steps to aid in the exploration and development of the modified implementation strategies for the adoption and enhancement of PDIS in the discharge process.

Ethics and dissemination Ethics approval for the study was obtained from the Joint Chinese University of Hong Kong-New Territories East Cluster Clinical Research
Strengths and limitations of this study

- An innovative approach with a structured multistage study design involving qualitative inquiry and Delphi expert discussion employing the theoretical domains framework, behavioural change wheel and realistic evaluation will be used to investigate the implementation of discharge summary for patient at hospital discharge.

- The findings enhance the understanding of the outcome and the pathway to reach the outcome of the implementation of a newly developed discharge information summary for patients.

- Apart from the traditional top-down approach in policy design, the involvement of implementation science facilitates decision making by systematically channelling opinions and suggestions from various stakeholders such as patient and caregivers to improve the quality of care and patient outcomes.

Ethics Committees. Results of the study would be released as a report submitted to the Health and Medical Research Fund of Food and Health Bureau of the Hong Kong Government. The result would also be published in international peer-reviewed medical journals and presented in conferences.

Trial registration number ChiCTR2000034382.

\section{BACKGROUND}

Hospital discharge is not the final step of the patient's journey. For holistic management of the patient's chronic morbidities, the patient is sent from secondary care back to primary care, including receiving services from formal or informal caregivers and community clinics. ${ }^{1}$ During the transition, common errors such as medication discrepancies may lead to adverse drug effects. ${ }^{23}$ Confusion and 
danger can easily arise among elderly patients and/or caregivers in different levels of care, particularly in the transition to home care after hospital discharge. ${ }^{4}$

Forster et al found that nearly one in five service users experienced an adverse event within 3 weeks following hospital discharge. These adversities ranged from serious laboratory abnormalities to permanent disabilities, and up to $66 \%$ were accounted for by drug-related problems and medication errors. ${ }^{3}$ Other sources of adversity included insufficient information transfer and inadequate follow-up in primary care. ${ }^{5}$ These effects might further lead to unplanned emergency department visits or hospital readmissions. ${ }^{6}$ In recent times, unnecessary service utilisation and readmission have become associated with poorly executed transitions and have added an extra burden to the health system. ${ }^{7}$ Thus, a clear summary of information regarding medication and later medical follow-up is expected to facilitate a seamless transition on discharge. Similar concerns are also found in the regular patient experience surveys in Hong Kong (HK), which highlights the patient experience of being provided with adequate information before discharged from hospital to home, including medication side effects, warning signals regarding medications and information given to caregivers that was relatively negative impression and receiving lower scores than other aspects of care. This has been consistently highlighted as an area for improvement in the past decade. ${ }^{8}$

Comprehensive discharge instructions that are understandable to patients and their caregivers are vital communication tools to facilitate healthcare professionals to transfer important messages to patients and their caregivers in order to enhance the quality and continuity of care, adherence to medical treatment, and to be attentive to the warning signals to seek timely support, ${ }^{9-11}$ however, delivery of discharge instructions is often rushed and patients frequently do not understand and grasp them, particularly medication management instructions. $^{12}{ }^{13}$ Improving discharge information dissemination may improve patients' health literacy of self-care and health outcomes and avoid unnecessary healthcare utilisation and cost. Therefore, a well-written information that is provided to patients at discharge is a supplement to verbal inpatient education instructions. Despite its importance, evaluation of the implementation of discharge information is scanty.

\section{Development of postdischarge information summary}

In HK, all public hospitals are categorised in seven geographical clusters by the HK Hospital Authority (HA). A new system was suggested and developed accordingly-postdischarge information summary (PDIS) in public hospital setting in 2017. The committee members and experts in the PDIS team together with the Information technology Department of HA codeveloped the new system on top of the current electronic medical records for piloting. It is expected to be an effective platform for enhancing information dissemination on discharge, reducing medication non-adherence and adverse effect and therefore improving the health status among patients. The PDIS intervention was launched at the Department of Medicine of the first four public acute hospitals between 2018 and 2019 from three geographical clusters: New Territories East, New Territories West, Kowloon Central and HK East. The healthcare staff in the concerned departments has received training regarding the new platform. The dissemination of PDIS target those aged 65 and above discharged by the Department of Medicine. On discharge, it is a mandatory provision that a nurse or a doctor should present and explain the written PDIS to the patient or caregiver at the geriatric and medicine ward. As PDIS involves information technology integration, the implementation of PDIS intervention involved the sequential rollout in four hospitals in different phases with non-randomised cluster steppedwedge design (figure 1). The order in which the hospitals

\begin{tabular}{|c|c|c|c|c|c|c|c|c|c|c|c|c|c|c|}
\hline Current & & & \multicolumn{12}{|c|}{2018} \\
\hline $\begin{array}{c}\text { Manpower } \\
\text { (Planned } \\
\text { Minimum } \\
\text { Sample) }\end{array}$ & NOV & $\mathrm{DEC}$ & JAN & FEB & MAR & APR & MAY & JUN & JUL & AUG & SEP & OCT & NOV & $\mathrm{DEC}$ \\
\hline $\begin{array}{l}\text { Hospital A } \\
\text { Doctor: } 84(10) \\
\text { Nurse: } 390(10)\end{array}$ & & & & & & & & & & & & & & \\
\hline $\begin{array}{l}\text { Hospital B } \\
\text { Doctor: } 119(10) \\
\text { Nurse: } 418(10)\end{array}$ & & & & & & & & & & & & & & \\
\hline $\begin{array}{l}\text { Hospital C } \\
\text { Doctor: } 84(10) \\
\text { Nurse: } 390(10) \\
\end{array}$ & & & & & & & & & & & & & & \\
\hline $\begin{array}{l}\text { Hospital D } \\
\text { Doctor: } 48(10) \\
\text { Nurse: } 207(10)\end{array}$ & & & & & & & & & & & & & & \\
\hline
\end{tabular}

Figure 1 Non-randomised stepped-wedge trial of implementation of postdischarge information summary (PDIS) in four hospitals: manpower of healthcare professionals and suggested minimum sample size for evaluation. 
received the intervention is determined by their readiness. Non-randomised stepped-wedge design allows us to compare the healthcare professional's view not only at different contextual environment but also at different time points and different length of time in the implementation. From the initial beneficial findings on patient experience, HA decided to scale up the PDIS to other geriatric and medicine wards at the remaining $23 \mathrm{HA}$ acute and rehabilitation hospitals.

\section{Implementation and components of PDIS intervention}

In the past discharge process, patients will receive a piece of discharge summary, follow-up appointment slips, referral forms for further investigation and prescription slips with QR codes for medication information. The patients can scan the QR code to display the effects and possible side effects of all medications. Since the list is very detailed and involves medical jargon, patients and their caregivers seldom check the list. In order to enhance the presentation of information for self-care management and to reduce adverse events due to medication errors during the transition state, an autogenerated PDIS has been introduced to replace the dissemination of piecemeal information in numerous documents in 2017. The PDIS provides comprehensive discharge information. This information includes three types of information essential for the transition care of patients: (1) the patient's personal information, including name, gender, age, diagnosis and discharged hospital; (2) a standardised salient medication reminder (SMR) highlighting the most relevant and important side effects and warning signs of prescribed medicines requiring attention after discharge; and (3) a list of all follow-up appointments in $\mathrm{HA}$, including the date, time, and venue of consultation and examination. The consensus SMR was developed using the Delphi method, in which an expert consensus conference was conducted to develop a framework for an effective discharge planning system incorporating side effects / warning signs of medication. ${ }^{14} 15$

According to a preliminary evaluation, those patients received the PDIS expressed positive experience of discharge information and their overall experience on receiving a clear and understandable medicine information, the sufficiency and helpfulness were also improved significantly $(\mathrm{p}<0.05)$ after the rollout of PDIS when compared with the intervention before. However, there are considerable disparities or variations regarding when the healthcare staff implemented PDIS in hospitals in different HA clusters. The preliminary staff survey revealed they had different perceptions on the positive outcomes from PDIS such as its design, implementation process and work efficient in their hospitals. The differences in individual and collective behaviour of healthcare staff in different HA clusters may be one of the critical factors influencing its implementation and effectiveness. Thus, systemic evaluation of the adoption of PDIS would help to provide more valuable information about its implementation and enhancement to the system in the future. Thus, the aim of this study is to evaluate the implementation of a PDIS by healthcare professionals and suggest a modified strategy for enhancing the implementation. Thus, the objectives of the study include:

1. To systematically identify barriers and facilitators from the perspective of healthcare professionals (doctors and nurses) to the adoption of PDIS with the theoretical domains framework (TDF).

2. To identify behaviour techniques or possible strategies to address the context-specific implementation issues of PDIS with the behavioural change wheel (BCW).

3 . To develop a new theory-based implementation strategy package for the enhancement of the full-scale intervention of PDIS in all inpatient setting, addressing modifiable facilitators at behavioural, organisational and policy levels using the Delphi expert discussion.

The findings would also help to standardise the implementation process and optimise the benefit of the intervention before scaling up.

\section{METHODS}

\section{Hypotheses of research study}

The hypotheses of the study are (1) there is a knowledge gap between PDIS design and the belief of healthcare professionals regarding PDIS implementation ; (2) there are different implementation strategies (behaviour) to deliver PDIS in the four pilot hospitals and (3) the modifiable facilitators at behavioural, organisation and policy levels can enhance to the full-scale intervention of PDIS.

\section{Study design and research objectives}

A multistage design with qualitative inquiry and Delphi expert discussion will be used to systematically explore the perceived barriers and enablers of the implementation of the PDIS in geriatric and medical care. The design goal is to enhance the understanding of the underlying mechanism of the behaviour variation considering internal and external contexts in the implementation of the PDIS programme, and to modify implementation strategies of adoption and enhancement of PDIS in the discharge process.

\section{Ethics approval, informed consent}

Ethics approval for the study was obtained from the Joint Chinese University of Hong Kong-New Territories East Cluster Clinical Research Ethics Committees. Written informed consent consisting of the study's aim, data collection procedure and participants' rights will be obtained prior to the commencement of the qualitative interviews and Delphi expert discussion.

\section{Patient and public involvement}

Patients or the public were not involved in the design, or conduct, or reporting, or dissemination plans of our research.

\section{Theoretical framework for systematic evaluation of implementation strategies}

Three theoretical frameworks will be adopted to guide data collection and analysis. First, the TDF is a well-established 
and validated comprehensive theoretical framework for the assessment of barriers and targeting resources to influence behavioural change for implementation projects. ${ }^{16}$ It consists of 84 theoretical constructs sorted into 14 domains: (1) knowledge; (2) skills; (3) social/professional role and identity; (4) beliefs about capabilities; (5) optimism; (6) beliefs about consequences; (7) reinforcement; (8) intentions; (9) goals; (10) memory, attention and decision processes; (11) environmental context and resources; (12) social influences; (13) emotion; and (14) behavioural regulation. ${ }^{17}$ Second, after confirming the influencing factors in the implementation, we will employ the BCW, an advice tool for providing a structured approach to designing or updating behavioural change interventions and strategies. ${ }^{17}$ It can be used to promote a systematic and comprehensive analysis of the available intervention options and to ensure that component parts of an intervention act synergistically. ${ }^{17}$ Third, the realistic evaluation framework (REF) is an enhanced theory-driven approach that can be used to understand the underlying mechanism of the behaviour variations considering the internal and external contextual settings. ${ }^{18}$ In summary, the TDF offers an integrated framework that enables identification of relevant factors influencing variations in staff and links to implementation strategies likely to bring about behavioural change using BCW and REF for further scaling of PDIS implementation in other hospitals and specialities.

\section{Participants}

Healthcare professionals include doctor and nurses who are working in the Departments of Medicine of the first four public acute hospitals which have launched PDIS since the pilot phase in 2018.

\section{Data collection}

To understand the impact of the implementation of enhanced discharge information-PDIS in terms of adoption, barriers, facilitators and suggestions for improvement in hospital settings-we will approach doctors and nurses who had been involved in the discharge process for at least 3 months in the corresponding hospital for in-depth individual interviews (step 1). They will be asked to provide comments and suggestions on staff training on PDIS, their roles and procedures for the implementation (explaining and handling queries from patient or caregivers), manpower arrangements among the work environments, hardware support and future development of PDIS. Step 2 will not involve participant recruitment as our research team attempted to map the findings in step 1 with theoretical models. In step 3, a purposive sampling of stakeholders in the implementation of PDIS in the setting will be employed. The PDIS project comprises doctors, nurses and administrative/management will be invited for the Delphi expert discussion.

The study consists of three step (figure 2) and details of each step are shown as follows:
Step 1: The goal of step 1 is to identify barriers and enablers to the adoption and enhancement of PDIS from an implementation perspective. Key informant interviews with doctors and nurses in four pilot HA hospitals will be conducted. The interview will explore the behaviours and perceptions of contextual factors, including organisational factors and policies that need to be changed to facilitate PDIS adoption and enhancement among interviewees. We will focus on two key aspects: (1) identifying who performs what behaviour, when, where, why and how; and (2) considering the contextual attributes of the target behaviour such as political atmosphere, policy, organisation culture and setting, action sequences, interdependence behaviour at the multidisciplinary team level, and patients' expectations. The TDF-informed interview guide will consist of open-ended questions from each of the 14 theoretical domains (figure 3) to elicit the first response, followed by a series of prompts to probe deeper into the interviewee's views. Some of the sample questions has been listed in online supplemental appendix 1 . This interview will allow us to form an in-depth understanding of stakeholders' perspectives on the complexity of implementing PDIS within the immediate context of a hurried discharge process as well as the broader internal and external sociocultural contexts of HA hospitals.

Based on the suggested guidelines, ${ }^{19}$ the authors will approach 6-10 participants with diverse experiences for an in-depth analysis in order to satisfy the concept of 'information power' for the qualitative interview. In order to have a rich and diverse sample from doctors and nurses, a minimum of 10 interviews will be conducted for each professional in each of the hospitals for the initial data analysis. According to principle of data saturation, additional interviews to would be conducted until no new themes emerged. ${ }^{19}$ Thus, the minimum number of interviews to be conducted will be at least 10 interviews $\times 2$ professionals $\times 4$ hospitals $=80$ (figure 1 ). An invitation of recruitment will be sent to all eligible staff. If more than 10 healthcare professionals in each profession from the corresponding hospital agree to participate in the interview, we will establish a sample pool from which to draw a random sample of sequential numbers. The research team further invite more healthcare professionals from the sample pool to conduct additional individual interviews to ensure data saturation if necessary. The individual interviews will be conducted in Cantonese through face-to-face interviews.

Step 2: After identifying the barriers and enablers in step 1 , theory-based implementation strategies comprising complex behavioural change techniques will be developed, with a specific goal of overcoming barriers and enhancing enablers in the adoption and enhancement of PDIS at individual, hospital (multidisciplinary team) and policy levels. These implementation strategies packages will be developed by a mapping approach, which is a systematic and formal process for tailoring strategies based on identified barriers to and enablers of change. ${ }^{20}$ In the mapping process, behavioural change techniques 
Step 1: Identification of barriers and modifiable facilitator from the perspectives of healthcare professionals (doctors and nurse)

Purpose: To adopt and enhance PDIS with Theoretical Domains Framework (TDF)

Methods:

Individual in-depth interview guided by Theoretical

Domains Framework (TDF) across healthcare

professionals

(Doctors: $n=48$ \& Nurses: $n=48$ )

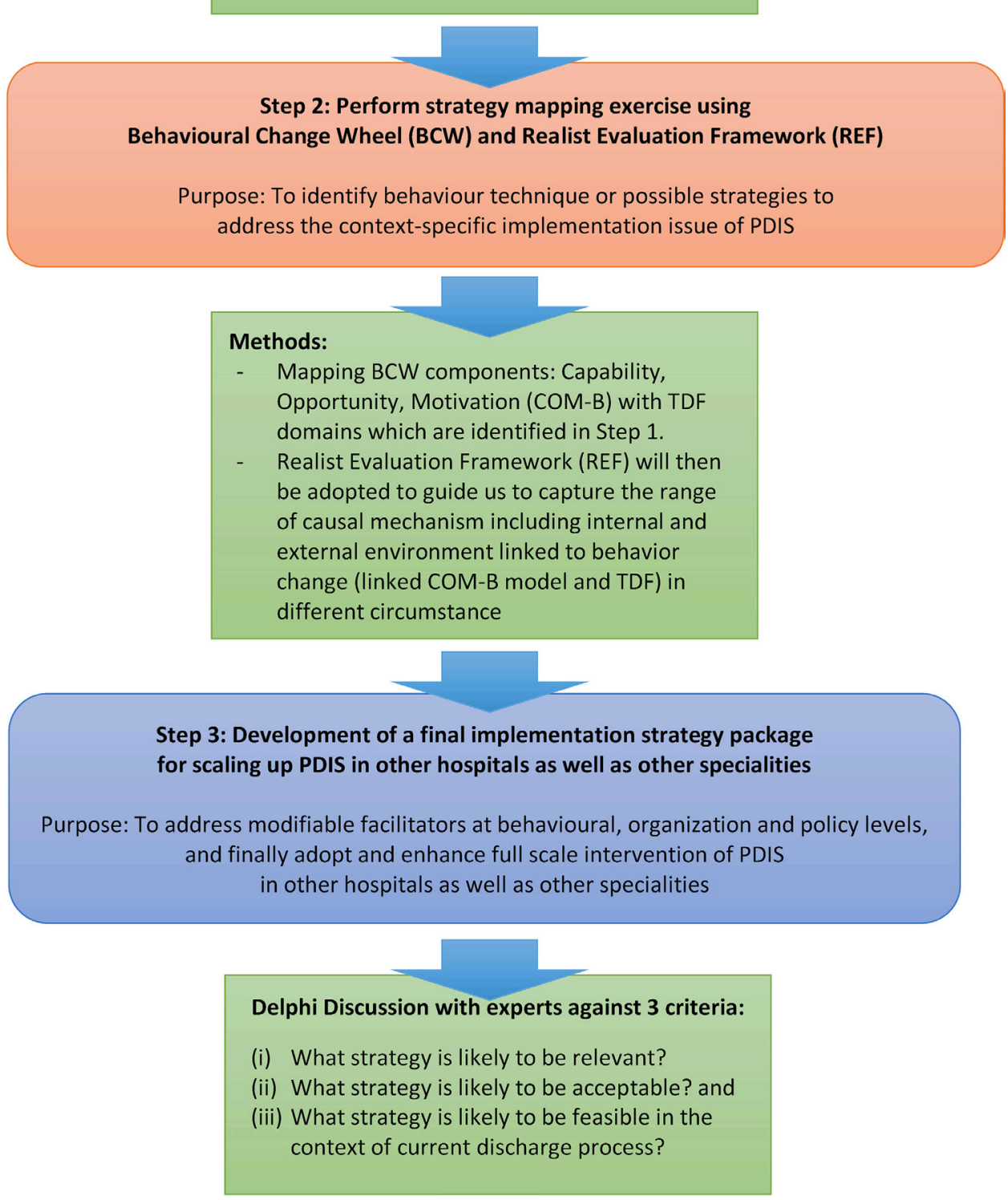

Figure 2 Study methodology. PDIS, postdischarge information summary.

from the BCW matrix will be selected to target the most important domains of TDF identified in step 1.

The BCW matrix V.1 consists of 93 effective behavioural change techniques in three components: capability, opportunity and motivation (COM-B), which potentially overcomes barriers and facilitates enablers in the implementation of the programme (figure 3). ${ }^{21}$ Having identified the relevant COM-B components in the relevant TDF domains, we will explore how to address barriers and strengthen enablers by focusing on specific implementation strategies, which is the red zone of BCW. Once the implementation strategies are developed, we will evaluate which policies are required to support the delivery of implementation strategies, which is the grey zone of $\mathrm{BCW}$. The mapping process requires a logical interpretation of what the behavioural target would be, and what components of the behaviour system would need to be changed to achieve that outcome. The categories in each 
Domains and Component Constructs in

Theoretical Domains Framework (TDF)

Informed Interview Guide
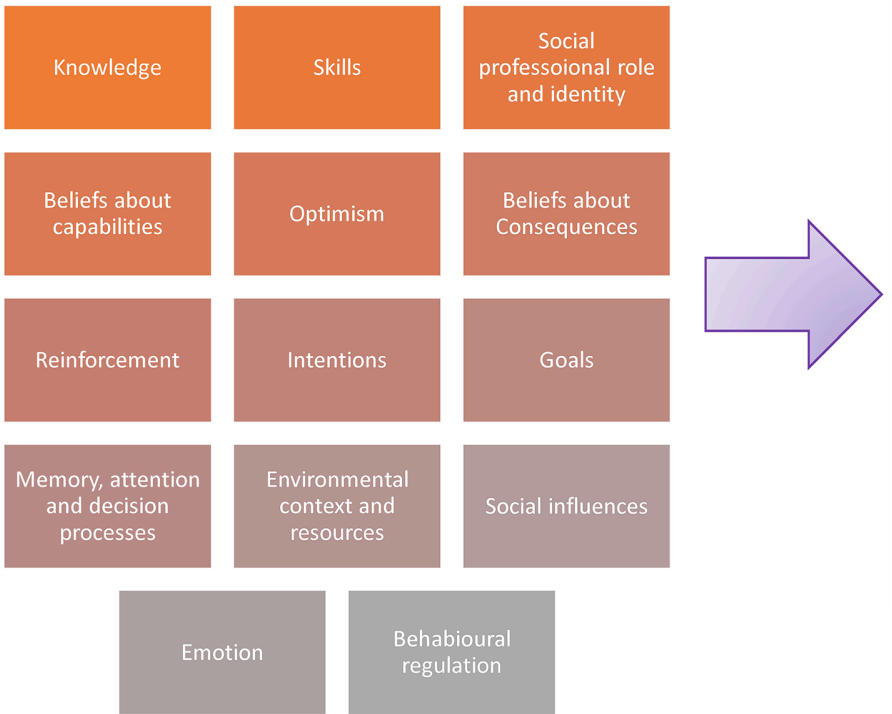

Source:

TDF - Atkins, L., Francis, J., Islam, R. et al. A guide to using the Theoretical Domains Framework of behaviour change to investigate implementation problems. Implementation Sci 12, 77 (2017).
Three Components of Behaviour Change Wheel (BCW) Guide Mapping Process between

Barriers/Facilitators and Behaviour Change

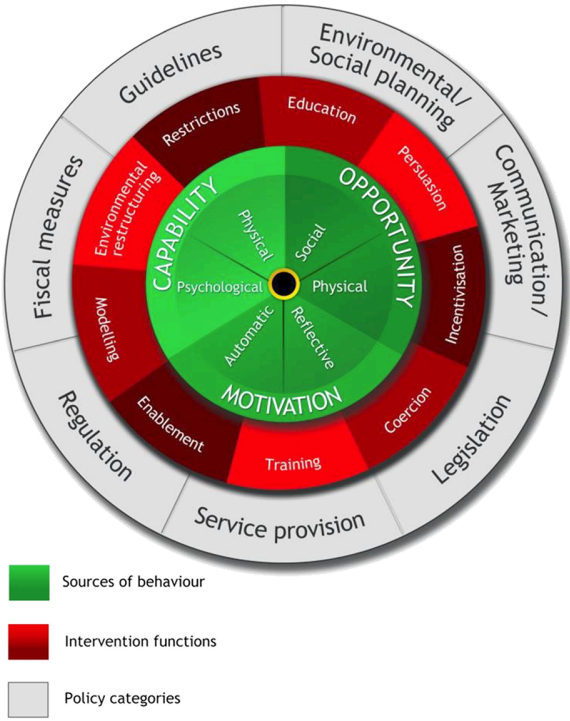

Source:

BCW - Michie, S., van Stralen, M.M. \& West, R. The behaviour change wheel: A new method for characterising and designing behaviour change interventions. Implementation Sci 6, 42 (2011).

Figure 3 Theoretical framework for data collection and analysis.

colour zone require an understanding of the mechanism, including the internal and external environment linked to the behaviour. The realist evaluation framework (REF) is adopted to enable us to capture the range of causal mechanisms that may be involved in behavioural change in certain circumstances. ${ }^{21}$ REF is a theory-driven evaluation approach commonly used in the programme evaluation in reality. ${ }^{18}$ It will be used to configure the behavioural change in the context of the study setting by knowing what behaviour works (outcome) produced by a causal mechanism (mechanism) in a given contextual environment (context). This outcome-mechanismcontext configuration enhances the understanding of implementation strategies across a range of different hospital cultures and political settings.

Step 3: We will use the findings from Steps 1 and 2 to develop a final implementation strategy package for scaling up PDIS in other hospitals as well as other specialities using the Delphi expert discussion. ${ }^{22}$ The development will involve discussions with experts using three criteria: (1) what strategy is likely to be relevant in HA hospitals and/or local usage (relevance); (2) what strategy is likely to be acceptable within the HA (organisational acceptance) and (3) what strategy is likely to be feasible in the context of the current discharge process (feasibility). This step is deemed to seek corporate-wide advice from the expert members to scaling up PDIS in other hospital as well as other specialities in real hospital setting. It will facilitate local adoption of modified implementation strategies for PDIS. The experts are required to rate the three criteria on a 5-point Likert scale with
$1=$ strongly disagree to $5=$ strongly agree. At least two rounds of Delphi expert discussion will be conducted, after which a final list of behavioural change techniques with high local relevance, acceptability, and feasibility will be generated. The implementation strategy is endorsed as a technique in the strategy package if $\geq 70 \%$ of experts rate 4 (agree) or 5 (strongly agree) for all three criteria based on agreement standards. The implementation strategy will not be included in the strategy package if $<70 \%$ of experts rated 4 or above for all three criteria. The experts are requested to comment or make suggestions for those rating $\leq 3$. For each strategy, the median rating for the three evaluation criteria will be calculated as it is less influenced by outliers when compared with mean values. The $\mathrm{SD}$ and $\mathrm{IQR}$ values for each strategy will also be calculated to reflect the magnitude of disagreement among experts. ${ }^{23}$

A purposive sampling strategy will be used to identify a balanced number of PDIS delivery stakeholders from doctors, nurses, and administrative/management representatives. The PDIS project team comprises 46 key stakeholders, including (1) the chairman and cochairman of the Central Committee of Complaint Management and Patient Engagement; (2) the Chairmen of the Geriatric Sub-committee of the Coordinating Committee in Medicine; (3) delegates of four pilot hospitals (doctor, nurse, pharmacist); (4) delegates of non-pilot hospitals; (5) HA representatives from the Central Nurse Division, Quality and Safety Division, and Chief Pharmacist's Office, Information Technology and Health Information; and (6) a representative from the patient experience project of the 
Chinese University of Hong Kong. Thus, the Chairman of the PDIS project team will nominate at least two PDIS project members from each of the categories $1-5$ to join the Delphi expert discussion with a minimum of 10 experts. Those who participated in the interviews in step 1 will not be eligible to join step 3 . The research team will thus act as an intermediate person to liaise the best implementation strategies between the front-line healthcare professionals and those in the managerial level.

\section{Data analysis}

A qualitative data analysis NVivo V.12 software will be used to code, organise, and manage the transcribed data to facilitate the data interpretation for step 1. Two independent reviewers will be responsible for data coding. Subsequently, an inductive coding approach will be used to generate subcategories of specific behaviour and its determinants within the initial coding scheme of the 14 TDF domains. Finally, the coded data will be further inductively examined in order to identify relevant theoretical domains for the target behaviour (adoption and enhancement of PDIS). Domains that are associated with specific themes or beliefs that would be considered as barriers or enablers to the adoption and enhancement of PDIS will be shortlisted. Domains in relation to contextual factors (context) and determinants (mechanism) will also be highlighted. These findings will allow us to design a BCW-based implementation strategies package with the consideration of the REF in step 2.

The coding in step 1 and initial mapping using BCW in step 2 will first be conducted by the research assistants under the supervision of investigators. To enhance the consensus among coders, we will train the coders to articulate their understanding of the coded text and provide a clear rationale for selecting a particular domain to increase the credibility of evidence for informing the decisions and to ensure researchers are perceiving the data in similar perspectives and frameworks. ${ }^{24}$ The findings from the mapping exercise in step 2 will then be discussed with investigators with knowledge in implementation science, behavioural change and programme knowledge. The reliability between the two coders will be evaluated by an inter-rater kappa score across all domains. Intercoder reliability would be considered satisfactory when the kappa score is $>0.6 .{ }^{25}$ Further consensus-making meetings and discussion among the study team will be arranged if this threshold is not reached. Thus, the investigators will discuss and decide the judgement of the coders to have consensus between the disagreements and aim to achieve joint decisions through systematic communication. The study team may introduce a third coder if necessary.

\section{DISCUSSION}

This study appears to prospectively assess healthcare workers' views on the implementation of supporting discharge information dissemination from different perspectives to enhance the quality of care at patient discharge. We propose an innovative study approach to link the implementation science and REF to guide the study design and bridge the knowledge gap of the quantitative data and the actual challenges faced by front-line healthcare workers. The traditional measurement by the questionnaire limits the vision and framework of the PDIS and its assessment. The TDF guided in-depth qualitative views collected from the healthcare workers will provide thoughtful ideas out of the vision of the research team. The realistic evaluation of qualitative data facilitates team members' understanding of what is happening, their learning and challenges, their problem-solving, and ultimately, the actions they take (or do not take) with regard to PDIS implementation. Furthermore, multiple rounds of Delphi expert discussions with stakeholders of the implementation of the PDIS project would enable us to have a more systematic channel for generating feasible and effective strategies into the strategic implementation of the PDIS instead of collecting individual responses for the suggestions. Thus, this study will identify PDIS implementation issues within the complex, busy, and dynamic hospital environment and how front-line healthcare workers deal with roadblocks and unexpected events, and describe the link between healthcare workers and implementation action in the discharge process. User feedback is essential to facilitate the early stage development of a brand-new idea in order to fulfil the needs of all stakeholders, include both front-line healthcare professionals who supply and explain the PDIS, and the patients and caregivers who receive the information. These findings will lead to new organisational strategies for managing multidisciplinary project teams and implementing information dissemination to patients at hospital discharge.

Despite these advantages, we anticipate a number of challenges when conducting the study. First, the qualitative interview will involve a large sample size and clinical engagement; in particular, doctors are frequently identified barriers in perspective-sharing research because of their tight schedule. We hope to address this challenge by engaging all levels of clinical staff from the start of the project with an explicit project aim to build rapport with the staff and enhance the buy-in idea. To increase the flexibility arrangement of data collection from the healthcare professionals, the study team will try to arrange interview times and locations convenient for them. Refreshment will also be provided as a token of appreciation for their efforts and time during their tight schedule. We will ensure that all the reported findings are anonymous and the suggested strategies are feasible in all participating hospitals to overcome their barriers for the PDIS implementation. Second, timely and accurate data collection for a large-scale, stepped-wedge study is challenging, especially during the COVID-19 pandemic. Thus, we will shift the setting to have telephone interview or video meeting for the interviews if necessary.

Furthermore, several limitations were identified in the study. The adoption of non-randomised clustered stepped-wedge design is proposed in the study due to 
the managerial and system readiness among the selected hospitals. The participants' feedback might be attributed with geographical or temporal factors. Understanding the participants' perception on barriers and facilitators would assist the consideration of the difference between clusters and the effect of the implementation. The views from the healthcare staff perspective on the PDIS implementation will be collected in the study but excluded those from patients or their caregivers. Thus, future evaluation studies should be conducted among patients or caregiver perspectives to investigate their experience and provide suggestions for optimising the PDIS after scaling up. Also, quantitative indicators such as readmission rate, patient experience, patient-reported outcome measures could be acted as outcome measurements to provide an objective evaluation on the intervention.

At the end of the study, we believe our study will facilitate clinical practise in a busy ward, improve patient experience and outcomes, and provide a model for organisation-wide quality improvement initiatives.

\section{Ethics approval and consent to participate}

Ethics approval for the study was obtained from the Joint Chinese University of Hong Kong-New Territories East Cluster Clinical Research Ethics Committees. Written informed consent consisting of the study's aim, data collection procedure and participants' rights will be obtained prior to the commencement of the qualitative interviews and Delphi expert discussion.

Acknowledgements The study is supported by Food and Health Bureau, The Government of Hong Kong Special Administrative Region (Reference no: 17180721). The financial support of the Centre for Health Systems and Policy Research is from The Tung's Foundation.

Contributors ELYW, EKY and KST were involved in conception and design of the study. DD and PM provided their professional advice on conducing qualitative study and planning for data analysis. ELYW is the lead author and prepared the article. AWLC and JCHL were involved in drafting the article. All the authors were involve in critical revision and in final approval of the article.

Funding Health and Medical Research Fund by Food and Health Bureau, The Government of Hong Kong Special Administrative Region.

Competing interests None declared.

Patient consent for publication Not applicable.

Provenance and peer review Not commissioned; externally peer reviewed.

Supplemental material This content has been supplied by the author(s). It has not been vetted by BMJ Publishing Group Limited (BMJ) and may not have been peer-reviewed. Any opinions or recommendations discussed are solely those of the author(s) and are not endorsed by BMJ. BMJ disclaims all liability and responsibility arising from any reliance placed on the content. Where the content includes any translated material, BMJ does not warrant the accuracy and reliability of the translations (including but not limited to local regulations, clinical guidelines, terminology, drug names and drug dosages), and is not responsible for any error and/or omissions arising from translation and adaptation or otherwise.

Open access This is an open access article distributed in accordance with the Creative Commons Attribution Non Commercial (CC BY-NC 4.0) license, which permits others to distribute, remix, adapt, build upon this work non-commercially, and license their derivative works on different terms, provided the original work is properly cited, appropriate credit is given, any changes made indicated, and the use is non-commercial. See: http://creativecommons.org/licenses/by-nc/4.0/.
REFERENCES

1 Jon Glasby RL, Ellins J, Davison D, et al. Understanding and improving transitions of older people: a user and carer centred approach, Southampton: NIHRSDO. United Kingdom: NHS National Institute for Health Research, 2010.

2 Williams H, Edwards A, Hibbert P, et al. Harms from discharge to primary care: mixed methods analysis of incident reports. $\mathrm{Br} J \mathrm{Gen}$ Pract 2015;65:e829-37.

3 Forster AJ, Murff HJ, Peterson JF, et al. The incidence and severity of adverse events affecting patients after discharge from the hospital. Ann Intern Med 2003;138:161-7.

4 Kripalani S, Jackson AT, Schnipper JL, et al. Promoting effective transitions of care at hospital discharge: a review of key issues for hospitalists. J Hosp Med 2007;2:314-23.

5 Mesteig M, Helbostad JL, Sletvold O, et al. Unwanted incidents during transition of geriatric patients from hospital to home: a prospective observational study. BMC Health Serv Res 2010;10:1.

6 Taché SV, Sönnichsen A, Ashcroft DM. Prevalence of adverse drug events in ambulatory care: a systematic review. Ann Pharmacother 2011;45:977-89.

7 Friedman B, Basu J. The rate and cost of hospital readmissions for preventable conditions. Med Care Res Rev 2004;61:225-40.

8 Hong Kong Hospital Authority. Patient experience survey on inpatient service 2017. Hong Kong, 2018.

9 Horstman MJ, Mills WL, Herman LI, et al. Patient experience with discharge instructions in postdischarge recovery: a qualitative study. BMJ Open 2017;7:e014842.

10 Preen DB, Bailey BES, Wright A, et al. Effects of a multidisciplinary, post-discharge continuance of care intervention on quality of life, discharge satisfaction, and hospital length of stay: a randomized controlled trial. Int J Qual Health Care 2005;17:43-51.

11 Shepperd S, Lanmin NA, Clemson LM. Discharge planning from hospital to home. Cochrane Database Syst Rev 2010;20:CD000313.

12 Chugh A, Williams MV, Grigsby J, et al. Better transitions: improving comprehension of discharge instructions. Front Health Serv Manage 2009;25:11-32.

13 Saidinejad M, Zorc J. Mobile and web-based education: delivering emergency department discharge and aftercare instructions. Pediatr Emerg Care 2014;30:211-6.

14 Wong EL-Y, Tang K-S, Cheung AW-L, et al. Development of salient medication reminders to facilitate information transfer during transition from inpatient to primary care: the Delphi process. BMJ Open 2021;11:e041336.

15 Yam CHK, Wong ELY, Cheung AWL, et al. Framework and components for effective discharge planning system: a delphi methodology. BMC Health Serv Res 2012;12:396.

16 Atkins L, Francis J, Islam R, et al. A guide to using the theoretical domains framework of behaviour change to investigate implementation problems. Implement Sci 2017;12:77.

17 Francis JJ, O'Connor D, Curran J. Theories of behaviour change synthesised into a set of theoretical groupings: introducing a thematic series on the theoretical domains framework. Implement Sci 2012;7:35.

18 Pawson R, Tilley N. Realistic evaluation. Thousand Oaks, CA, US: Sage Publications, Inc, 1997: xvii, 235.

19 Francis JJ, Johnston M, Robertson C, et al. What is an adequate sample size? operationalising data saturation for theory-based interview studies. Psychol Health 2010;25:1229-45.

20 Michie S, Richardson M, Johnston M, et al. The behavior change technique taxonomy (V1) of 93 hierarchically clustered techniques: building an international consensus for the reporting of behavior change interventions. Ann Behav Med 2013;46:81-95.

21 Michie S, van Stralen MM, West R. The behaviour change wheel: a new method for characterising and designing behaviour change interventions. Implement Sci 2011;6:42.

22 Boulkedid R, Abdoul H, Loustau M, et al. Using and reporting the delphi method for selecting healthcare quality indicators: a systematic review. PLoS One 2011;6:e20476.

23 Holey EA, Feeley JL, Dixon J, et al. An exploration of the use of simple statistics to measure consensus and stability in delphi studies. BMC Med Res Methodol 2007;7:52.

24 O'Connor $\mathrm{C}$, Joffe $\mathrm{H}$. Intercoder reliability in qualitative research: debates and practical guidelines. Int J Qual Methods 2020;19:160940691989922.

25 Landis JR, Koch GG. The measurement of observer agreement for categorical data. Biometrics 1977;33:159-7. 